\title{
SHIELDING EFFICIENCY OF A FABRIC BASED ON AMORPHOUS GLASS-COVERED MAGNETIC MICROWIRES TO RADIATION EMITTED BY A MOBILE PHONE IN 2G AND 3G COMMUNICATION TECHNOLOGIES
}

Simona MICLĂUŞ* simo.miclaus@gmail.com

George MIHAI** george.mihai@ancom.org.ro

Angel Marian ARON** angel.aron@ancom.org.ro

Cristian MITRESCU** cristian.mitrescu@ancom.org.ro

Paul BECHET* pbechet@gmail.com

Octavian BALTAG*** octavian.baltag@bioinginerie.ro

* “Nicolae Bălcescu” Land Forces Academy, Sibiu, Romania

** National Authority for Management and Regulations in Communications - ANCOM, Bucharest, Romania *** TerrafluxControl, Iaşi, Romania

\begin{abstract}
A dual band mobile phone model was used to check the shielding properties of an amorphous ferromagnetic textile against the radiation emitted by the handset. Two frequencies belonging to the $2^{\text {nd }}$ and $3^{\text {rd }}$ generation of mobile emission technologies were used, $897 \mathrm{MHz}$ and $1950 \mathrm{MHz}$. The specific absorption rate (SAR) of energy deposition in a human head phantom was measured in standardized conditions. The textile contained micrometric-diameter wires of a ferromagnetic mixture embedded in a thin glass coat and weaved in a specific way. A set of fabric orientations and configurations (layering) were provided in the experiment in order to achieve a better shielding to the phone's radiation. Compared with the non-shielded handset, SAR deposited in the head while using the fabric-covered phone could be decreased up to $30 \%$ of its initial value - in case of $2 G$ technology and up to $24 \%$ - in case of $3 G$ technology. This type of material shows one of the highest shielding efficiencies of the electric-field component in near-field exposure conditions reported until now. A cubic curve of SAR decrease in depth of the head was revealed in both uncovered and covered handset, the effect of shielding being larger at the higher frequency.
\end{abstract}

KEYWORDS: mobile phone, SAR, near-field, electromagnetic shielding, magnetic amorphous material 


\section{Introduction}

Solutions for an efficient protection of the user against the radiation emitted by a mobile phone were continuously investigated, so that literature on this subject was published starting with the first years after 2000. A brief review of the proposed shielding solutions and of the materials used for radiation reduction is presented in (Miclăuş et al., 2016). Materials such as conductive meshes, conductive fabrics, conductive foams, wiremesh screens, multi-layers, ferrites, resistive sheets and metamaterials were all checked for their efficiency. Simulations were also conducted at microwave frequencies so as to emphasize for example the differences due to particular geometries of the metallic wires in a textile or their weaving density when a wire-meshed material was used as a shield (Rosu, Druta \& Baltag, 2016). In general the results concerning field reduction showed that shielding efficiency greatly depends on a series of variables: position of the material with respect to the handset's antenna, structure and composition of the material, orientation of the absorbing/reflecting fibers with which emitted radiation interacts, dimensions of the entire shield, etc. (Tomovski, Grabner, Hungsberg, Kallmeyer \& Linsel, 2011; Wessapan, Srisawatdhisukul \& Rattanadecho, 2011; Dutta, Jayasree \& Srinivasa, 2016; Miclaus et al., 2016). Both a decrease of the specific absorption rate (SAR) of energy deposition in the head exposed to a "shielded" mobile phone can be achieved, but sometimes even an increase of local SAR could be observed. The highest reduction percentage of SAR ever reported when using a shielding solution for a mobile phone was of $60 \%$ (Miclaus et al., 2016) but few such results were generally reported, while the average percentage of SAR reduction was usually situated at around $15 \%$ from the value obtained when using the mobile handset as it is - with any shield.
The subject of mobile phone radiation reduction is of continuous interest, especially for the safety of sensitive persons, hard users and children. The use of fabrics based on amorphous magnetic microwires for SAR reduction was scarcely studied to date, as of our knowledge, but their shielding properties at microwave frequencies were carefully studied (Makhnovskiy \& Panina, 2006; Yong-jiang, et al., 2007; Liberal, 2013; Devkota et al., 2014; Baranov, Larin \& Torcunov, 2017). Therefore we proposed here a preliminary empirical study in which single or multiple layers of such a material to be used as a cover of the mobile handset and then tracing SAR modifications in a phantom human head. By using a standardized SAR measurement procedure we determined the SAR reduction factor due to the presence of the fabric in a set of experimental configurations. The shielding effect was assessed for just one model of mobile phone which was used consecutively in two communication standards: $2^{\text {nd }}$ generation (GSM) technology and $3^{\text {rd }}$ generation (UMTS) technology respectively. The preliminary results indicate that this material type provides significant radiation attenuation in both frequency ranges, fact that entitles further future research on this subject.

\section{Materials and Methods}

SAR is a measure of tissue heating when radiofrequency (RF) radiation propagates thru it. SAR depends on both local internal E-field strength $\left(E_{\text {int }}\right)$ and on the dielectric properties of the tissue. On the other hand, the connection between SAR and heating rate $(\Delta T / \Delta t)$ is made thru the specific heat capacity of the tissue, $c$. Therefore, the ways to express SAR are:

$$
S A R=\frac{\sigma * E_{i n t}^{2}}{\rho}=c * \frac{\Delta T}{\Delta t} \quad(\mathrm{~W} / \mathrm{kg})
$$

Where $\sigma$ is the electric conductivity of the tissue and $\rho$ is its masic density.

The normative reference used for present SAR measurements in the phantom 
of the human head when it is exposed to RF field emitted by the mobile phone antenna was standard "Recommended Practice for Determining the Peak Spatial-Average Specific Absorption Rate (SAR)" (IEEE $1528,2013)$. The handset was tested in the "cheek" position. The earpiece of the handy was always in contact to the ear or to the fabric covering the handset respectively. The COMOSAR-SATIMO measurement system was used and it functioned in a shielded room where all experiments took place. SAR distributions in the human head phantom could be observed by area and zoom scans, and values of average or peak SAR could be calculated by the OpenSAR software. European legislation indicates averaging of SAR on $10 \mathrm{~g}$ of equivalent tissue that is why we will refer mainly to this parameter. SAR_10g value should not exceed $2 \mathrm{~W} / \mathrm{kg}$ in the human head when using a phone, for safety reasons (ICNIRP, 1998). The 6-axis robot measurement system of COMOSAR-SATIMO enabled fine movement of the miniature electric (E)-field probe to scan the volume of the head in 27 measurement points, very fast (steps of $8 \mathrm{~mm}$ on each direction). This method was used in the present approach for just coarse assessment of SAR reduction capabilities of the amorphous magnetic fabric.

A mobile phone model Samsung GT-I9195I was used in all the experiments. The phone was used in two emission situations: a) a signal specific to $2 \mathrm{G}-$ GSM900 communication technology a time division multiple access (TDMA) scheme was initiated (crest factor $=8$ ) at the frequency $\mathrm{fl}=897.6 \mathrm{MHz}$; b) a signal specific to $3 \mathrm{G}$ - UMTS communication technology - a wideband code division multiple access (WCDMA) scheme was initiated (crest factor $=1$ ) at the frequency $\mathrm{f} 2=1950 \mathrm{MHz}$. In each case the call was established at the maximum output power with the base station simulator.

At frequency $\mathrm{fl}$, the liquid simulating human brain filling the head phantom had the next dielectric parameters: real and imaginary part of the relative permittivity were $\varepsilon^{\prime}{ }_{\mathrm{r}}=41.5, \varepsilon{ }_{\mathrm{r}}=19.4$ and the electric conductivity was $\sigma=0.97 \mathrm{~S} / \mathrm{m}$. At frequency $\mathrm{f} 2$, the dielectric parameters of the liquid were: $\varepsilon_{\mathrm{r}}^{\prime}=40, \varepsilon{ }_{\mathrm{r}}=12.93, \sigma=1.4 \mathrm{~S} / \mathrm{m}$.

SAR in the head was measured initially with no material covering the handset, and this measurement was called REF since it was used as a reference SAR value. Then the amorphous magnetic material was used as a cover on the surface of the handset and the contact was maintained with the phantom's head during measurements. All the time the phone was in touch with either phantom's shell directly or indirectly, by inserting the textile. The fabric cover was used in 1, 2 or 4 layers respectively while the direction of the weaving yarns was positioned in 2 ways: normal (A) or rotated by 90 degrees (B). Finally, shielding cases were called 1A, 1B (meaning one layer of material in normal and then in rotated position was used as a cover); 2A, 2B; 4A, 4B.

The fabric used in current measurements is based on glass-coated amorphous ferromagnetic wires twisted together with cotton or synthetic (kapron) yarns. The chemical composition of the amorphous wire is $\mathrm{Co}_{68} \mathrm{Mn}_{7} \mathrm{~B}_{15} \mathrm{Si}_{10}$. The diameter of the microwires ranges between $(10-13) \mu \mathrm{m}$, and the outer diameter of the glass insulation ranges between $(15-20) \mu \mathrm{m}$. The main charecteristics of the fabric were presented in Baltag et al., (2000) and Rau, Iftemie, Baltag \& Costandache (2011).

\section{Results and Discussion}

Figure no. 1 presents examples of SAR values distribution on a surface containing peak SAR value, when the phone was used in GSM standard, while Figure no. 2 refers to the same situations but when phone was used in UMTS communication standard. Each first image called "REF" in both figures refers to the 
unshielded phone, while the other three situations refer to single or double layered shielding fabric applied over the handset surface. We notice that in some cases, multilayers do not necessarily conduct to a drop in SAR values. Also, in some cases (GSM900 - situations 2B, 4A, 4B), the OpenSAR software detected, at the surface scanning, a secondary hot spot comparable to the main one (in these cases, 2 values of SAR have been computed and finally it has been reported just the higher value).

For a proper comparison of the situations we used the SAR value averaged over $10 \mathrm{~g}$ of equivalent brain tissue. In Figure no. 3 we represented SAR_10g values in the phantom head for all investigated cases (six cases in which amorphous magnetic shielding was used and one without shield, REF) when $2 \mathrm{G}$ communication signal was emitted by the phone. In all situations when the material covered the phone, a reduction of the average SAR was gained. The highest SAR reduction was obtained when just one layer of material was used and when the orientation of its fibers was "B" - in that case SAR_10g was reduced 3.3 times from its value when the phone was uncovered. Interesting to note is that using 2 or 4 layers of material didn't improve shielding, but contrary.
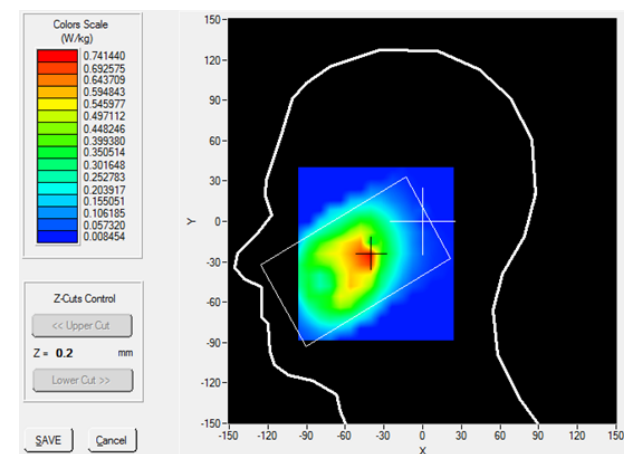

REF
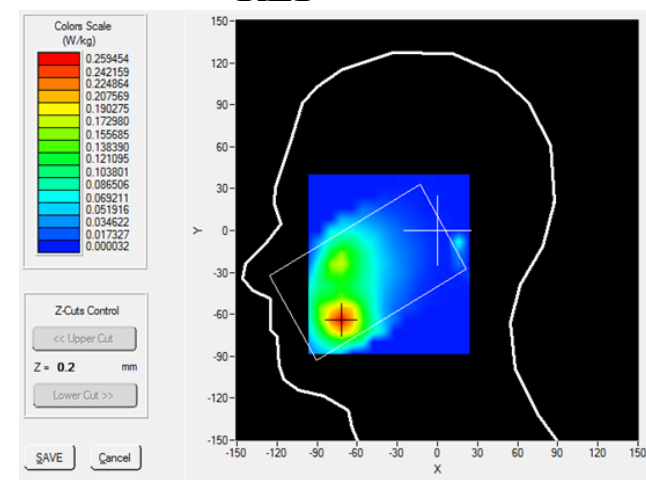

1B
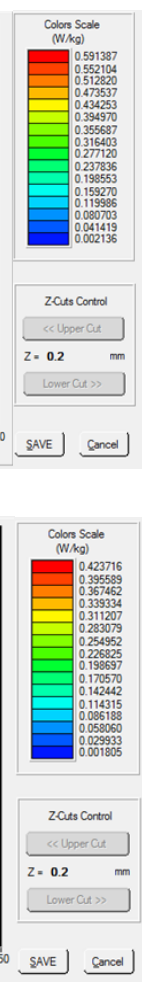

SAVE] Sencel

\section{B}

Figure no. 1: Surface distribution of SAR in the phantom head while using mobile phone in GSM emission technology: REF is for phone without any cover, $1 A$ and $1 B$ make use of one layer of amorphous ferromagnetic fabric set in two orthogonal directions, $2 B$ makes use of double layered fabric in one of the fibers orientations

In Figure no. 4 SAR_10g reduction can be observed when the phone emitted $3 \mathrm{G}$ signal. In this case, significant SAR reductions were obtained with "B" orientation in all cases, and by using multiple layers of fabric we gained even better reduction capabilities in some cases. The highest SAR reduction factor was of $1 / 4$ from the SAR value of unshielded handset. "A" orientation of the shielding 
fabric was in all cases less efficient than "B" orientation.

Table no. 1 synthesizes the reduction factors of SAR_10g when using the amorphous magnetic microwires textile as a cover. Maximum percentage of SAR_10g reduction in the material in case of GSM emission was of $70 \%$, and in case of UMTS emission was of $76 \%$. Such significant reductions of the radiation in a material have rarely or never been reported before.

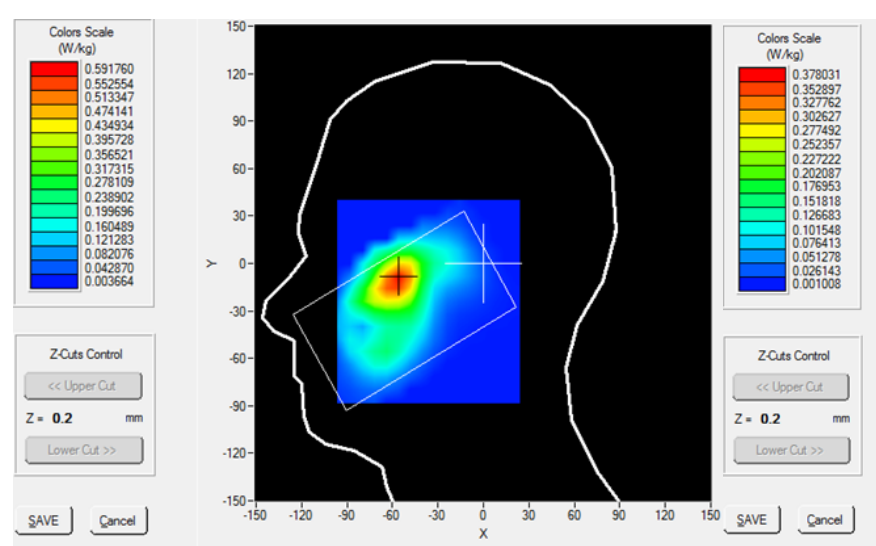

REF

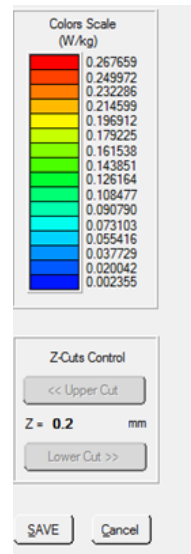

SAVE] cancel]

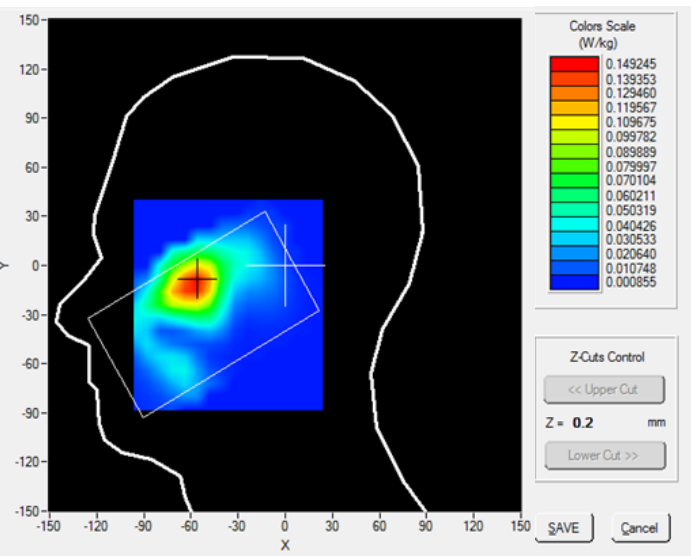

1B

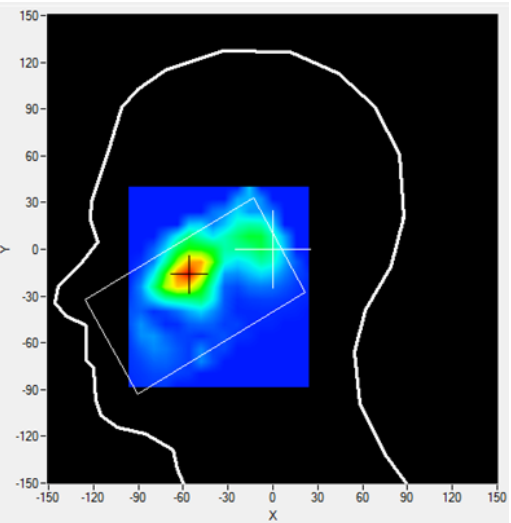

1A

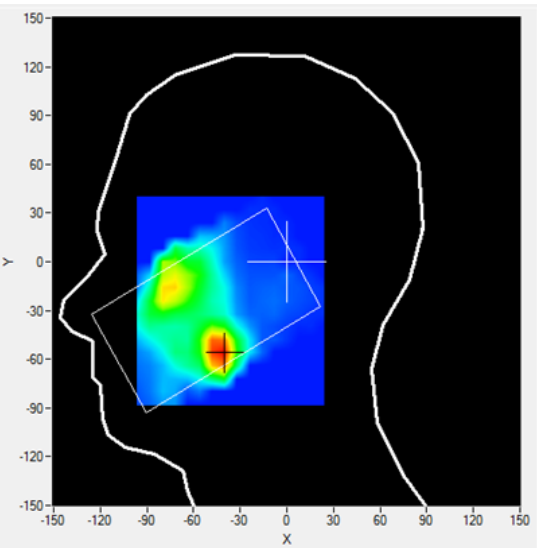

2B

Figure no. 2: Surface distribution of SAR in the phantom head while using mobile phone in UMTS emission technology: REF is for phone without any cover, $1 A$ and $1 B$ make use of one layer of amorphous ferromagnetic fabric set in two orthogonal directions, $2 B$ makes use of double layered fabric in one of the fibers orientations

Figure no. 5 and Figure no. 6 indicate the curves of SAR attenuation in depth of the head phantom for GSM and UMTS signals respectively. By comparison between uncovered phone (REF case) and shielded phone by a single layer fabric (cases 1A and 1B), one observes that decreasing rate may be different. At least in case of higher frequency of UMTS signal, local slopes of SAR decrease with distance are different in each case. However, all SAR attenuations follow a $3^{\text {rd }}$ degree polynomial regression curve which is printed on the graphs, while the coefficient of determination is $\mathrm{R}^{2}=1$. This behavior indicates that the fabric introduces 


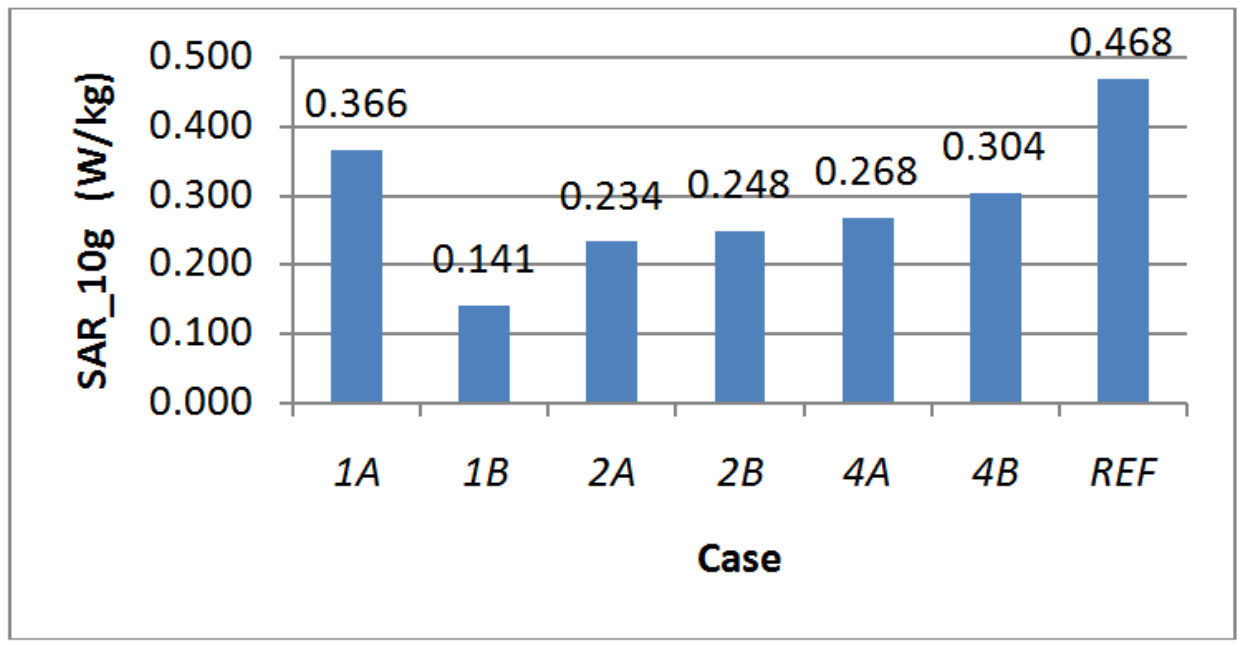

Figure no. 3: Average SAR values for GSM emitted signal at $f 1=897.6 \mathrm{MHz}$ in case of unshielded handset (REF) and in case of different shielding situations

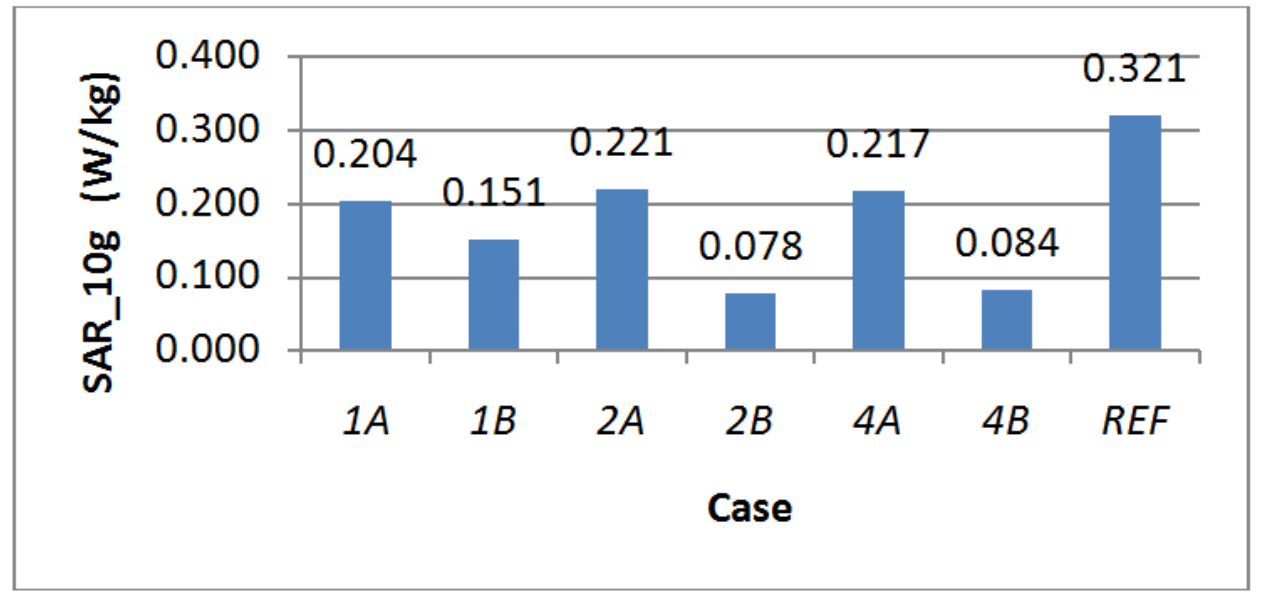

Figure no. 4: Average SAR values for UMTS emitted signal at $f 2=1950 \mathrm{MHz}$ in case of unshielded handset (REF) and in case of different shielding situations

Table no. 1

Factors of SAR reduction due to amorphous magnetic shield presence reported to the case when the handset was unshielded

\begin{tabular}{|c|c|c|}
\hline $\begin{array}{c}\text { Fabric } \\
\text { Covering/ } \\
\text { case no. }\end{array}$ & $\begin{array}{c}\text { GSM emission/ } \\
\text { Factor of SAR_10g reduction }\end{array}$ & $\begin{array}{c}\text { UMTS emission/ } \\
\text { Factor of SAR_10g reduction }\end{array}$ \\
\hline 1A & 0.78 & 0.64 \\
\hline 1B & 0.30 & 0.47 \\
\hline 2A & 0.50 & 0.69 \\
\hline 2B & 0.53 & 0.24 \\
\hline 4A & 0.57 & 0.66 \\
\hline 4B & 0.65 & 0.26 \\
\hline
\end{tabular}


different volumic shielding coefficients mainly at frequencies whose wavelength tend to approach the texture dimensions of the magnetic fibers. In this regard, simulations would indicate more precisely the "rule" of improved shielding capabilities.

Based on the SAR results and by applying relation (1), we can calculate the duration a call might be prolonged with, by using the magnetic amorphous shield, for the same temperature increment in the head: for the GSM phone the call length may be increased 3.33 times and for UMTS phone the call length may be increased 4.17 times. These values, of more practical significance for a user, indicate the shielding efficiency range of this material.

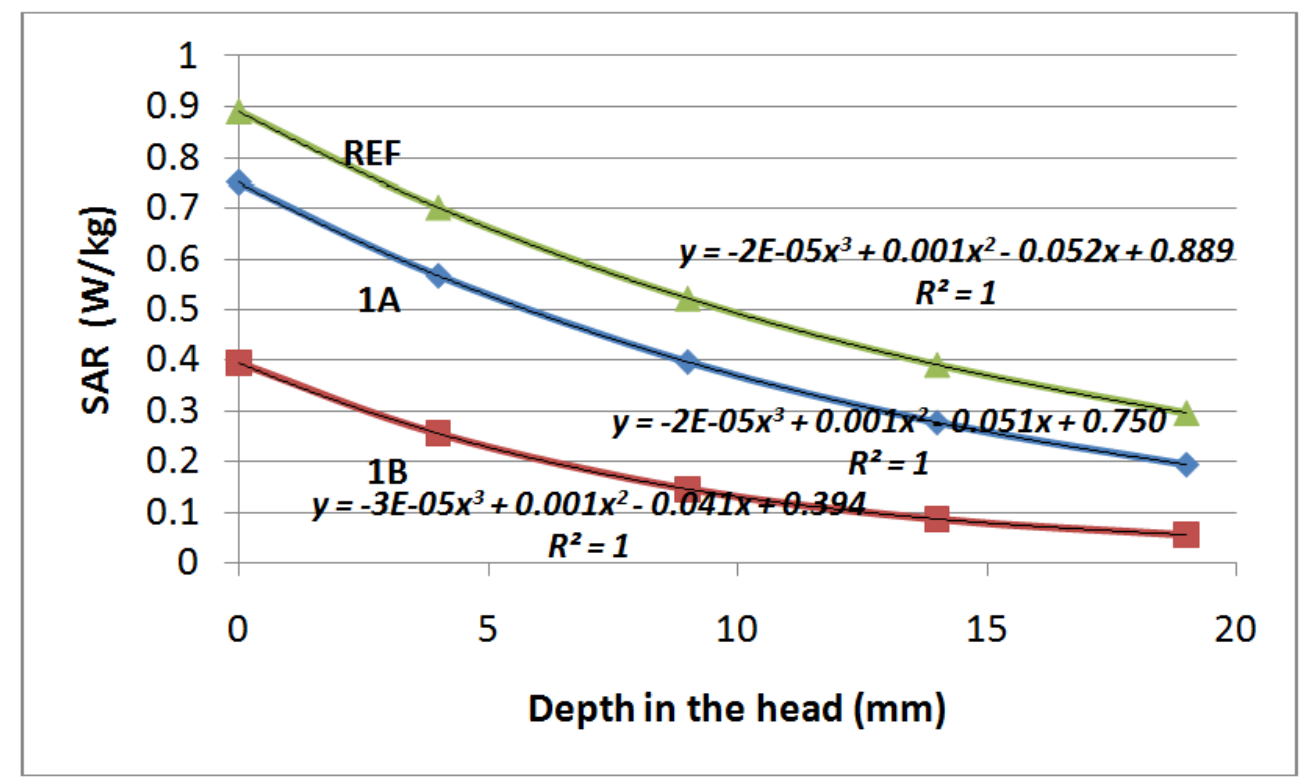

Figure no. 5: SAR attenuation with depth in the phantom head in case of GSM signal for unshielded (REF) and one-layer fabric shielded handset $(1 A, 1 B)$

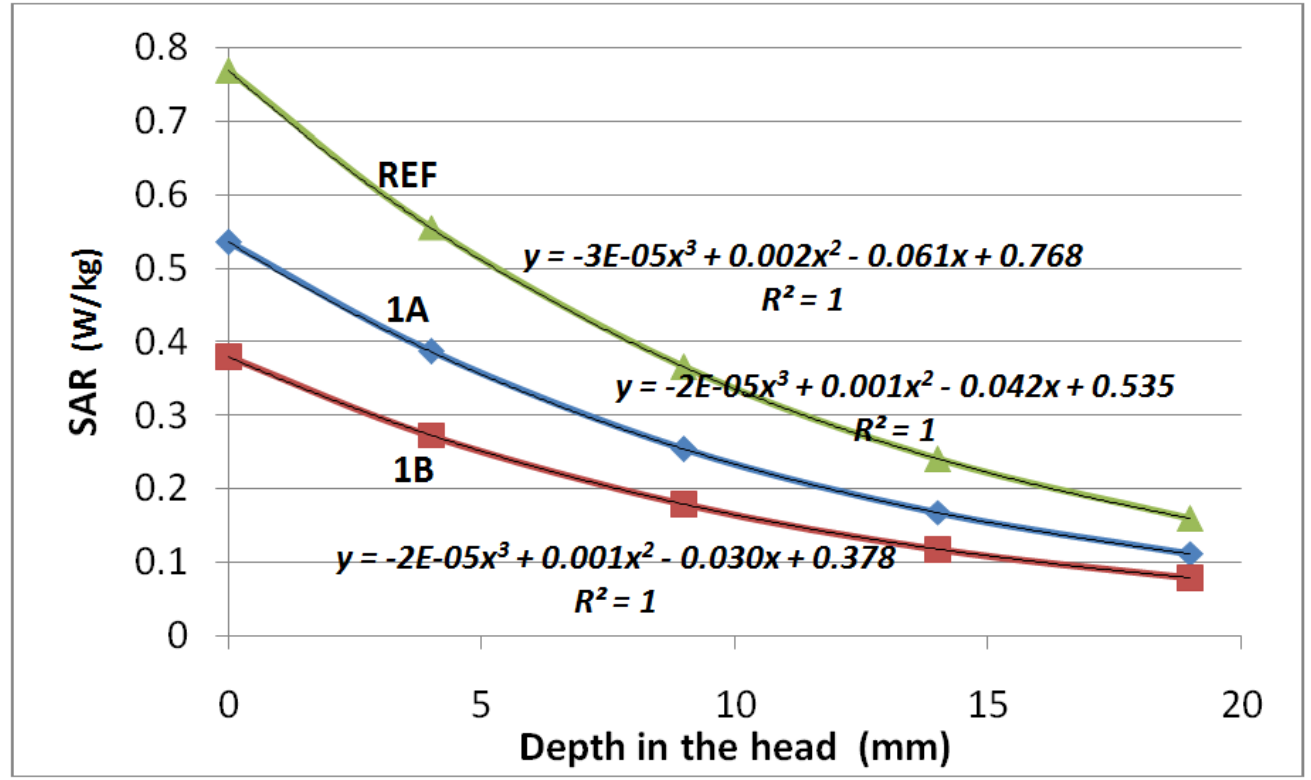

Figure no. 6: SAR attenuation with depth in the phantom head in case of UMTS signal for unshielded (REF) and one-layer fabric shielded handset $(1 \mathrm{~A}, 1 \mathrm{~B})$ 


\section{Conclusion}

One phone model was investigated by a standardized procedure in order to report the specific absorption rate of energy deposition in a human head phantom while using the phone at its highest emission power - either in direct contact to the head or covered by a material with electromagnetic shielding properties. The study was a pure preliminary experimental approach of the shielding capabilities of an amorphous ferromagnetic textile fabric used to decrease human exposure to mobile phone radiation. While inserted in the near field zone of antenna, the material, containing micrometric dimensions magnetic wires covered by thin glass, was checked in two positions and with three thickness values (by layering) for its SAR reduction properties when it covered the handset. Since modern phones make use of both $2 \mathrm{G}$ and $3 \mathrm{G}$ communication technologies, two frequencies of these technologies were investigated.

It was revealed that this material has exceptional shielding properties on the electric-field component at investigated frequencies of $897 \mathrm{MHz}$ and $1950 \mathrm{MHz}$ and needs further study. The SAR reduction percentage due to the fabric presence on the handset surface was of maximum $70 \%$ if reported to SAR value of the unshielded phone functioning in GSM standard and of $76 \%$ in UMTS standard. Layering the fabric is not more efficient generally, but rather the position and orientation of the microwires relative to the handset antenna plays an important role in shielding.

\section{REFERENCES}

Baltag, O., Cantore, C., Costandache, D., Haras, S., Larin, V., \& Torcunov, V. (2000). Study of a texture of an amorphous magnetic material able to screen non-ionizing radiation, Non-linear Electromagnetic Systems, (eds.) Di Barba, P., \& Savini, A., IOS Press, 717-720.

Baranov, S. A., Larin, V. S., \& Torcunov, A. V. (2017). Technology, Preparation and Properties of the Cast Glass-Coated Magnetic Microwires, Crystals 7, 136.

Devkota, J., Colosimo, P., Chen, A., Larin, V. S., Srikanth, H., \& Phan, M. H. (2014). Tailoring magnetic and microwave absorption properties of glass-coated soft ferromagnetic amorphous microwires for microwave energy sensing, Journal of Applied Physics, 115, 17 A525.

Dutta, P. K., Jayasree, P. V. Y., \& Srinivasa Baba, V. S. S. N. (2016). SAR reduction in the modeled human head for the mobile phone using different material shields, HumanCentric Computing and Information Sciences, 6, 3.

ICNIRP. (1998). Guidelines For Limiting Exposure To Time-Varying Electric, Magnetic And Electromagnetic Fields (Up To 300 GHz), Health Physics, 74 (4), 494-522.

IEEE 1528. (2013). Recommended Practice for Determining the Peak Spatial-Average Specific Absorption Rate (SAR) in the Human Head from Wireless Communications Devices: Measurement Techniques.

Liberal, I. (2013). Amorphous Glass-Coated Ferromagnetic Wires in Microwave Engineering, Ph. D. Thesis, Department of Electrical and Electronic Engineering, Public University of Navarra, Department of Electrical and Electronic Engineering, Pamplona, Spain.

Makhnovskiy, D. P. \& Panina, L. V. (2006). Experimental demonstration of tunable scattering spectra at microwave frequencies in composite media containing CoFeCrSiB glasscoated amorphous ferromagnetic wires and comparison with theory, Physical Review B, Condensed matter, 74(6). 
Miclăuş, S., Bechet, P., Paljanos, A., Mihai, G., Pătru, I., Aron, A. M., \& Baltag, O. (2016). Shielding Effectiveness Of Some Conductive Textiles And Their Capability To Reduce The Mobile Phones Radiation, Proceedings of the $23^{\text {rd }}$ International Conference Knowledge-Based Organization, Vol. XXII, No 3, 524-530.

Rau, M., Iftemie, A., Baltag, O., \& Costandache, D. (2011). The Study of the Electromagnetic Shielding Properties of a Textile Material with Amorphous Microwire, Advances in Electrical and Computer Engineering, 11(1), 17-22.

Rosu, G., Druta, N., \& Baltag O. (2016). The Study of the Microwave Shielding Properties of Various Screen Configurations, Proceedings of the International Conference on Communications (COMM), Bucharest, 9-10 June 2016.

Tomovski, B., Grabner, F., Hungsberg, A., Kallmeyer, C., \& Linsel, M. (2011). Effects of electromagnetic field over a human body, SAR simulation with and without nanotextile in the frequency range 0.9-1.8 GHz, Journal of Electrical Engineering, vol. 62, no. 6, 349-354.

Yong-Jiang, D., Jian-Jun, J., Gang, D., Bin, T., Shao-Wei, B., \& Hua-Hui, H. (2007). Magnetic and microwave properties of glass-coated amorphous ferromagnetic microwires, Transaction on Nonferrous Metal Society of China, 17, 1352-1357.

Wessapan, T., Srisawatdhisukul, S., \& Rattanadecho, P. (2011). The effects of dielectric shield on specific absorption rate and heat transfer in the human body exposed to leakage microwave energy, International Communications in Heat and Mass Transfer, 38, 255-262. 